\title{
Le réel entre mimesis et transgression dans Jérémie ! Jérémie! de Dominique Fernandez
}

\author{
Michel Magniez \\ Lycée Jean Racine de Montdidier
}

Dominique Fernandez, élu à l'Académie française en mars 2007, est l'un des écrivains contemporains les plus présents sur la scène littéraire française. Le roman Jérémie! Jérémie!, est paru en 2005 chez Grasset et a été réédité par la Librairie générale française en 2007. Ce roman raconte le voyage de Fabrice Jaloux, un jeune étudiant parisien passionné par Alexandre Dumas, sur l'île d'Haïti. Engagé bénévolement dans une mission humanitaire internationale, il y recherche, dans le cadre de son DEA, les traces des ancêtres d'Alexandre Dumas. Il se rendra compte peu à peu que ses amis et lui-même ont été manipulés 
et ont participé en réalité, par leurs diverses activités sur l'île, à l'établissement d'un complexe hôtelier lucratif.

Le traitement du réel dans Jérémie! Jérémie! se caractérise par l'ampleur des fluctuations du roman entre, d'une part, l'esthétique du vrai, c'est-à-dire le constat de «ce qui est», et, d'autre part, la volonté de transgresser le réel, de chercher d'autres formes de réalité qui ouvrent de nouvelles perspectives sur le monde.

Il s'agit donc de déterminer comment Dominique Fernandez, par le biais de son jeune narrateur Fabrice, tente d'évoquer le plus fidèlement possible la réalité haïtienne, aussi bien dans sa dureté que dans sa beauté. Mais le réel dans Jérémie! Jérémie! ne se réduit pas à un simple compte rendu d'observations de voyage, si proches de la réalité soient-elles. Bien au contraire, Fernandez offre diverses modalités de transgression du réel: les rites et les cérémonies vaudous, d'une part, et le recours aux arts d'autre part, qui participent tous deux d'une expérience individuelle et collective de redécouverte du réel.

\section{La dureté du réel : les conditions de vie (et de mort) en Haïti}

Le roman de Dominique Fernandez est tout d'abord une observation sans concessions de la réalité haïtienne, dans sa dureté et dans sa crudité les plus poignantes.

Les bidonvilles de Port-au-Prince sont des exemples paradigmatiques de l'espace haïtien où, dans la narration de Fabrice, se révèle la pauvreté du pays : «Carrefour, le bidonville à la sortie de la capitale, effroyable chaos de taudis, d'ordures, d'humains en guenilles et de cochons vautrés dans les 
immondices $^{1} »($ p. 104). À cette description fait écho, quelques pages plus loin, l'évocation de Cité Soleil : «le bidonville le plus étendu, le plus populeux de Port-au-Prince.» (p.122) Le réel est alors offert au lecteur par le biais de l'accumulation d'éléments constitutifs du quotidien de l'île, et les détails relevés par le narrateur remplissent le cadre du paysage jusqu'à saturation du réel: "Les taudis, les masures en carton, les tentes en peau de chèvre s'écrasaient au fond de la baie jusqu'à la limite marquée par les flots. » (p. 122)

La description du réel se fait dans l'espace comme dans le temps. Le personnage-narrateur établit une terrible comparaison entre les circonstances de vie actuelles des Haïtiens et la période pourtant révolue de l'esclavage :

En quoi les Haïtiens vivaient-ils mieux qu'au temps de l'esclavage? Leurs conditions semblaient avoir empiré au contraire, car autrefois, [...] le maître avait intérêt à garder en bonne santé ceux dont il avait besoin pour s'enrichir. Aujourd'hui, le paludisme [...], la dysenterie [...], le sida [...] se développent sans obstacle ni frein sanitaire. (p. 100-101).

La dénonciation de la misère actuelle se double alors d'une mise en perspective du monde contemporain, par comparaison avec le passé.

C'est à la réalité économique et politique que se consacre essentiellement le narrateur, en se livrant à une violente critique de l'action des pouvoirs publics : «état calamiteux des routes, situation sanitaire désastreuse, carence de l'équipement et de l'encadrement scolaires, insuffisance de la lutte contre l'analphabétisme, non-assistance à des populations sans

\footnotetext{
${ }^{1}$ Les références des pages indiquées renvoient à l'édition originale parue chez Grasset en 2005.
} 
ressources» (p. 233). La vie quotidienne y apparaît extrêmement difficile en raison du manque absolu de sécurité, de jour comme de nuit :

[M. Schmidt] nous déconseillait de faire un pas dehors, même pour nous dégourdir les jambes. La nuit, cela allait sans dire : dès la chute des ténèbres [...], une obscurité sinistre enveloppe la ville. Faute d'éclairage public, chaque rue se change en coupe-gorge. Mais le jour aussi, sortir était dangereux. [...] Chacun avait une arme dans sa poche, et faisait peu de cas de la vie humaine. (p. 103)

La vie humaine n'a alors plus grande importance et la menace constante d'un danger imminent, qui plane dans ces conditions sur tout être humain, est à la fois l'expression d'une réalité quotidienne et un effet romanesque efficace pour maintenir le lecteur en haleine.

Le narrateur s'oblige peu à peu, dans ce cadre, à découvrir ce qu'est l'envers du réel, en décryptant autour de lui les symboles de la corruption. Dans le village de Jérémie, Fabrice se fait par exemple sensible à l'inscription dans l'espace de la collusion entre les pouvoirs financier, religieux et exécutif :

Le poste de police occupait un local minuscule au coin de la place Alexandre Dumas, non loin de l'unique banque de Jérémie. Église, police et banque n'étaient séparées que de quelques mètres. La concentration des trois pouvoirs au même endroit nous dispensait de rêver à de meilleurs jours pour la justice. (p. 265)

Le réel s'exprime dans la structure même de l'espace, qui révèle pour qui sait le lire les enjeux de pouvoir et les relations opaques entre les différents acteurs des mondes publics et privés. 
Cette évocation de la réalité haïtienne permet alors au narrateur de dénoncer l'action conjointe des autorités locales et des grands groupes étrangers. Pour que les investisseurs étrangers puissent exploiter de nouvelles terres, les habitants originels sont expropriés : «La hâte mise aux préparatifs disait assez les raisons de ce déménagement [...]. Le chatoiement des madras ne cachait pas la misère de l'exode.» (p. 211) Le narrateur dénonce alors la volonté d'installer un complexe touristique sur les côtes d'Haïti, et d'y exploiter le peuple sous couvert de fausses bonnes intentions: "Vos hôtels ne profiteront qu'à la Suisse, aux banquiers et aux hommes d'affaire de Lausanne, [...] vos activités se dérouleront en circuit fermé, [...] pas un dollar n'en reviendra aux habitants...» (p. 250-252)

Les réalités économique, politique et sociale sont donc dépeintes avec force détails. Mais le réel, en Haïti, ne se limite pas à ces observations désespérées, sinon désespérantes. La réalité d'Haïti apparaît comme dotée d'une bien plus grande complexité.

\section{La beauté du réel, ou la volonté de décrire tout le réel}

Le narrateur montre la multiplicité du réel par la superposition et la confrontation d'une réalité à une autre: Haïti n'est pas seulement une terre dévastée, c'est aussi un territoire riche et enivrant.

La description de la nature met en valeur « ces vallons émaillés d'une innombrable variété de fleurs, de lianes, de capillaires, de racines aériennes. De minuscules oiseauxmouches émeraude s'envolaient devant nous» (p. 205). Le 
narrateur, qui adopte sur ses propres convictions un regard critique rétrospectif, précise :

Haïti me paraissait encore cette terre presque chimérique, conforme à l'imagerie du paradis antillais: une nature opulente, un climat tropical mais tempéré par le voisinage de la mer, une population accueillante, aux mœurs douces et amicales, des fruits, des légumes en abondance. (p. 200-201)

Le narrateur joue lui-même sur les stéréotypes liés à la géographie physique d'Haïti dans le but de les condamner, mais il distingue de la manière la plus précise possible, d'une part, ce qui ressortit au cliché et à "l'imagerie» kitsch et snob du «paradis antillais » et, d'autre part, ce qui appartient à la réalité-même de lî̂le, exprimée par exemple dans cette courte phrase bâtie sur le rythme de deux octosyllabes consécutifs : « La terre haletait déjà de chaleur et de volupté. » (p. 132)

Le peuple haïtien lui-même, en lien constant avec le monde qui l'entoure, est caractérisé par sa paradoxale joie de vivre :

La gaieté, ou le courage d'être gais, semble être la vertu naturelle des Haïtiens. La précarité de leur vie quotidienne ne les empêche pas de rire, de chanter, de mordre dans chaque journée avec la même avidité que dans ces fruits somptueux que leur fournit un climat [...] secourable. (p. 99)

Ces notes du héros-narrateur ne laissent pas de surprendre par l'assertion qui les sous-tend: "rire», « chanter», profiter de l'instant sont les caractéristiques de ce peuple qui semble donc totalement détaché de la réalité si terrible qu'il doit affronter. Pourtant, l'harmonie entre ce peuple et le réel ne peut être plus parfaite puisque leur façon d'être-au-monde (« une aptitude personnelle au bonheur ») les relie pleinement à l'abondance et à la luxuriance que leur offrent la nature et le climat. 
Pour décrire ce qu'il voit, le narrateur ressent donc le besoin de coller au réel de la manière la plus véridique, la plus transparente possible. Fabrice s'interroge sur sa manière de percevoir et de retranscrire le réel :

Ne crains-tu pas de donner [...] dans le mythe du bon sauvage? [...] Non, concluais-je, je rejette tes objections. Je n'ai gobé aucune coquecigrue d'Occidental épaté par le dépaysement, la couleur, le contraste avec la grisaille bourgeoise. Je n'ai fait que constater ce qui est. (p. 145)

Fabrice se refuse à faire de l'île une description caricaturale, qui se contenterait de reprendre les poncifs et clichés occidentaux. Il rejoint en cela l'ambition et les interrogations présentes au cœur des nombreux récits de voyage écrits par Fernandez². L'auteur s'ingénie ainsi à établir une relation complexe de proximité, sans identification possible, pourtant, entre son personnage et lui-même.

Qu'il s'agisse de la misère et de la pauvreté, ou de la beauté et de la joie de vivre, c'est donc le réel sous toutes ses formes que veut peindre Fabrice Jaloux. On note cependant dans le roman un glissement progressif dans l'irréel,

\footnotetext{
2 Dans son Dictionnaire amoureux de la Russie, Fernandez réfute, par exemple, la vision traditionnelle de Saint-Pétersbourg en «Venise du Nord» (périphrase également utilisée parfois pour désigner Bruges et Amsterdam) : «La Venise du Nord? Non, non et non! Venise est un dédale de ruelles tortueuses, Saint-Pétersbourg un damier d'avenues rectilignes. Venise se découvre peu à peu, au fil des promenades, Saint-Pétersbourg se donne d'emblée. La beauté de Venise est pittoresque, la beauté de Saint-Pétersbourg est métaphysique » $(2004$, p. 630) De même, après avoir rappelé que «dans l'imaginaire des peuples», Rome est une ville "perçue comme lourde», il montre la fausseté de cette image et s'interroge, dans le Voyage d'Italie: «Alors? Où est la vérité de Rome? Dans l'obéissance ou dans la rébellion? Cette ville qu'on dit lourde n'a-t-elle pas dû se donner du lest pour combattre une propension innée à la légèreté? » ([1997] 2007, p. 697)
} 
l'insaisissable, le mystérieux ${ }^{3}$, qui semblerait venir contredire cette ambition de décrire strictement « ce qui est».

\section{Le vaudou, une échappée dans l'irréel}

La découverte du vaudou va être pour Fabrice l'occasion d'entrer dans un monde qui, jusque-là, lui était inconnu et de s'interroger sur une autre réalité.

Les certitudes de Fabrice sont peu à peu remises en cause par une série d'observations qui viennent bouleverser ses propres représentations mentales. Cette évolution apparaît dès le message que Fabrice envoie à Karine, sa petite amie, alors qu'il est bloqué à Fort-de-France, sa dernière escale avant Haïti :

Peut-être, écrit Fabrice, y-a-t-il au-dessus de nous, évoluant dans l'invisible, des esprits dont nous écartons l'influence par peur d'être menés où ils veulent nous conduire, dans la zone où règne une lumière si belle, si pure, qu'elle marquerait la fin du vouloir-vivre. (p. 95)

Il se distingue ainsi de Karine elle-même, dont il perçoit peu à peu la mentalité bourgeoise et matérialiste - dénuée de toute angoisse ou de toute recherche de spiritualité - , ainsi que celle de sa propre mère, qui, elle, est totalement «ignorante des forces profondes qui nous gouvernent à notre insu » (p. 67).

À l'opposé de celles et ceux qu'il avait côtoyés jusque-là, il verra s'accomplir en lui une véritable métamorphose. Il tirera profit de son voyage en Haïti en se découvrant lui-même : «Si je pars, écrit-il à Karine, c'est pour me quitter. Non pas pour

3 « Ce pays a une âme », déclare par exemple Boris, l'ami le plus proche de Fabrice, mais « je ne saurais définir ce qu'elle est » (p.171). 
m'oublier, remarque bien, mais pour me découvrir autre que je croyais que j'étais» (p.95). Cette transformation n'aura lieu que grâce à l'observation du monde qu'il découvrira : « ce pays m'obligera à voir les choses et à me voir moi-même d'une autre façon. Ce que je devine qui y subsiste des vieux rites africains correspond à mes interrogations du moment. » (p. 96)

Le mystère dont s'entoure l'île d'Haïti est palpable à chaque instant, notamment au travers des personnages qui l'habitent et qui mettent la terre en relation avec les dieux et cérémonies des cultes vaudous. Délira, dont le nom évoque les raras ${ }^{4}$ et la fureur religieuse, ressemble à une déesse de la Terre nourricière et des forces naturelles: «De ses membres alanguis, une telle force d'amour émanait, que les feuilles du mapou [...] se mettaient à frémir, et qu'autour de nous l'après-midi [...] cessait de paraître torride. [...]. La perfection de son entente avec le monde me stupéfiait. » (p.162) Narcisse Éphémère, au nom symbolique à plus d'un titre, est un adorateur du Dieu Ogoun-Badagri, "le seigneur des batailles, le dieu de la foudre et du tonnerre». Il montrera plusieurs fois à Fabrice les objets rituels de son culte : « les cailloux, les plumes de coq et la noix de coco ». Fabrice écrit à son sujet : « les forces qui l'agitaient de l'intérieur, étant arrivées à un degré supérieur d'intensité, demandaient à manifester leur domination » (p. 216). Narcisse est donc à la croisée de deux mondes : il est à la fois ancré dans la réalité tangible et dominé par des «forces» mystérieuses dont Fabrice ne peut parler plus en détail car elles lui sont totalement inconnues.

\footnotetext{
${ }^{4}$ Les raras sont des fêtes spontanées et improvisées de danse et de musique, qui se déroulent au son des tambours et peuvent " dégénérer en violences » (voir sp. 242 à 245)
} 
Le culte vaudou s'accompagne en particulier de plusieurs légendes au sujet des dieux, des morts et des vivants, dont l'une d'elles nous est ainsi racontée par Narcisse Éphémère : «Les gens y pensent qu'un mort il a pas envie de les quitter. Il a si peur de la solitude qui l'attend de l'aut'côté, qu'y veut à toute force retourner chez lui. » Fabrice complète ainsi cette évocation des légendes liées au vaudou : «Sans les ruses qu'on met en œuvre pour lui brouiller le chemin du retour, le mort s'échapperait la nuit du cimetière et reviendrait, pour l'entraîner dans sa tombe, jeter son dévolu sur un parent. » (p. 258-259). Il n'est nullement anodin que cette légende soit racontée à Fabrice, dont le père, mort alors qu'il était enfant, a fait sa réapparition dans sa vie peu de temps auparavant, lorsque le héros a appris que ce dernier, contrairement à ce qu'il avait toujours cru, n'avait pas lutté aux côtés des Républicains espagnols au temps de la guerre civile, mais avait été partisan des Franquistes ${ }^{5}$. L'insaisissable et le légendaire viennent ici rejoindre le réel historique et individuel le plus douloureux.

La description de la cérémonie vaudou à laquelle Narcisse prend part est l'un des moments forts du récit :

5 On se reportera avec intérêt à Ramon, la biographie consacrée par Dominique Fernandez à son propre père, pour saisir les liens spéculaires qui, mutatis mutandis, peuvent exister, dans Jérémie ! Jérémie !, entre le narrateur Fabrice et l'auteur lui-même. Dominique Fernandez s'est en effet lui-même longtemps interrogé sur l'évolution politique de son père, figure énigmatique qui, après avoir été membre de la SFIO et sympathisant communiste, adhéra au Parti Politique Français en 1937 et collabora avec le régime de Vichy. «Confusion, décidément, confusion, écrit Fernandez, dans le cœur de celui qui essaye d'y voir clair, à soixante-seize ans comme à quinze. » (Fernandez, 2009, p. 22) 
De plus en plus vite voltigeaient les doigts, de plus en plus vite crépitaient les baguettes, de plus en plus sauvagement Narcisse se laissait emporter par le tourbillon. Il tournait et dansait, dansait et tournait. Virevoltes sans trêves ni répit, possession de plus en plus complète, abandon progressif de son identité. $\mathrm{Au}$ fil des minutes, il se vidait de lui-même, saisi, dominé, ravi par une force supérieure. De même qu'un homme enfourche son cheval, le serre entre les cuisses et le plie à son vouloir, le dieu descendait sur lui et le contraignait à obéir. Je me rappelai ce que Narcisse m'avait dit : vassal d'Ogoun-Badagri, il devait se soumettre au seigneur des batailles, comme à un cavalier sa monture. (p. 219)

Les notations liées aux perceptions visuelles et auditives de la cérémonie viennent se mêler, dans un tourbillon d'actions et de perceptions décrites par une belle hypotypose, à l'apparition d'une «force supérieure » et du Dieu Ogoun-Badagri, dont la présence vient annihiler l'identité réelle de Narcisse. Le divin prend alors la place de la réalité sensible et palpable, et «le seigneur des batailles» s'affirme au travers de Narcisse Éphémère comme une réalité à part entière.

«La perception de mes sens, écrit Fabrice, s'était accrue. C'était comme si un nouveau sens m'était né. » (p. 236) De fait, la présence des mystères révélés par le vaudou exacerbe la perception du réel dont Fabrice est capable et fait disparaître alors la frontière entre le réel et l'irréel. Le narrateur est alors plus sensible au mystère du monde, de la nature et de luimême :

Il y avait comme un grand secret qui pesait sur la terre, dont j'étais seul à supporter la charge. À qui avouer ce dont j'ignorais moi-même la nature? De qui prendre conseil? J'interrogeai en vain le ciel, les arbres, les pierres blanches qui dessinaient un cercle autour de la mare asséchée. (p. 240-241) 
Alors qu'il comptait autrefois sur les autres pour diriger le sens de sa vie, qui était à l'avance tracé et dirigé par les conventions sociales et universitaires, c'est vers la Nature que se tourne Fabrice à ce moment du récit pour comprendre un secret dont il est «seul à supporter la charge». C'est cependant en s'interrogeant lui-même qu'il pourra résoudre bon nombre de mystères qui donnent naissance à son angoisse, mais cette démarche ne se fera que par le truchement de l'art et de l'écriture.

\section{Les arts : une herméneutique du réel par le biais de l'irréel}

Les arts sont pour Fabrice le moyen par excellence de répondre aux interrogations qui l'assaillent, parce qu'ils ouvrent, par le biais du voile et de l'illusion, une autre porte sur le réel.

La peinture apparaît plusieurs fois dans le roman, notamment par la description de deux tableaux du peintre italien Le Caravage, peintre auquel Dominique Fernandez a déjà consacré un ouvrage, intitulé La Course à l'abîme (2003). Les deux tableaux mentionnés dans Jérémie! Jérémie! sont Le Martyre de Saint Mathieu et David et Goliath, qui ont une double valeur prophétique puisqu'ils annoncent, par leurs sujets respectifs, la mort du peintre (rappelée par le narrateur), mais aussi la mort du héros lui-même, Fabrice, qui sera tué par un brigand appelé « Le Gamin ».

L'art pictural permet également de faire entrer le lecteur dans un monde fantasmagorique, comme le montrent les tableaux de Narcisse Éphémère : «Il peignait sous l'inspiration des forces de l'au-delà. L'acte de peindre, acte magique pour lui, le faisait communiquer avec les "esprits" (esprits de mort, esprits de vie) » (p.115). Ces tableaux représentent plusieurs êtres surnaturels, 
dont le sorcier Baron Samedi, «chef des esprits de la mort » et le Dieu Ogoun-Badagri (p. 114-115), « divinité belliqueuse attendue et redoutée » (p. 181). On apprendra que ce dieu, loin d'être une création de l'esprit de Narcisse Éphémère, est en fait la représentation stylisée et fantasmatique du général Dumas, père d'Alexandre Dumas, à la recherche duquel Fabrice est venu en Haïti.

Parallèlement à la peinture, la sculpture est aussi présente grâce à la statue de Pauline Bonaparte réalisée par Canova. Fabrice observe cette œuvre à la villa Borghese. Cette statue « noble et distinguée » (p. 41) déjoue en fait les pièges de la censure impériale et montre, par des détails qui ne sont visibles qu'aux fins observateurs, la véritable nature de la princesse. Par un effet de mise en abyme, la sculpture souligne dans le roman à quel point l'art dévoile le réel par le code et l'illusion.

La musique donne au roman une troisième perspective. Elle apparaît notamment par l'intermédiaire de M. Schmidt, qui dirige et finance la mission humanitaire dans laquelle Fabrice s'est engagé, et qui voue une grande admiration à Mozart (p. 33, $77,245)$. M. Schmidt a pris l'habitude d'associer à chaque jour de l'année les œuvres composées par Mozart ce jour-là, deux siècles auparavant. Fabrice relate ainsi l'une de ses rencontres avec ce personnage troublant: «M. Schmidt nous salua avec cordialité [...] Commençons, dit-il [...] C'était le jour, deux cent trente ans après, des Six danses allemandes, Köchel 509, dont il nous sifflota la gigue. » (p. 245) La chronologie réelle se double ainsi d'un écho aux œuvres de Mozart, qui fait de chaque jour une temporalité présente et la date de naissance d'une œuvre d'art créée dans une réalité passée. 
Mais Haïti apparaît tout entière comme une terre sauvée par le chant et la musique, qui permettent aux habitants d'échapper à leurs conditions de vie. Fabrice le rappelle ainsi : "On riait, on chantait, on dansait dans chaque maison, dans chaque case, si miteuse qu'en fût l'installation.» (p.145) La réalité musicale et artistique non seulement fait oublier, par sa beauté et sa jovialité, la réalité sociale et misérable de l'île, mais elle prend surtout le pas sur elle jusqu'à la remplacer dans le cœur des habitants et dans les choix d'écriture du roman. Elle fait partie intégrante de la représentation du réel haïtien.

La littérature marque enfin le point de départ et l'aboutissement de l'œuvre : le roman tout entier est marqué par l'empreinte d'Alexandre Dumas, dans son sujet, dans sa construction et dans le texte-même, puisque c'est une citation de Dumas qui termine le livre. On prendra comme exemple emblématique la relation d'identification qui s'élabore entre les deux personnages principaux du roman, Fabrice et Boris, et deux personnages de La Reine Margot, Hyacinthe de la Mole et Annibal de Coconnas, "les deux héros fictifs inventés par Dumas » (p. 196). L'identification à ces personnages parcourt le récit et met au jour la relation d'amitié qui s'instaure entre Boris et Fabrice. Les deux héros connaîtront d'ailleurs une fin tragique en Haïti, exactement semblable à celle des héros de Dumas dans la France du XVIe siècle.

On remarquera que Fabrice n'intervient pas en tant que créateur dans les arts plastiques et musicaux mentionnés. Il n'en est que le spectateur ou l'auditeur, en n'offrant qu'un regard ou une écoute, dont il saura tirer profit, envers ces manifestations artistiques. C'est cependant dans l'écriture qu'il parviendra à s'accomplir réellement - sa mort, conjointe à 
celle de Boris, marquant aussi bien la fin de son récit (qui serait impossible, si le romancier s'en tenait aux strictes lois du réel, puisque le narrateur semble mourir au moment même où il rédige ses dernières lignes) que la réalisation de son être et de ses aspirations profondes.

La dernière page du roman met enfin en évidence le lien irréfragable qu'entretiennent le réel et la littérature. Elle offre tout d'abord au lecteur une dernière citation de La Reine Margot (inscrite en exergue), qui donne de manière ambiguë l'explication de la mort des héros de Jérémie! Jérémie! et réaffirme le parallèle entre les héros de Dominique Fernandez (Fabrice et Boris) et ceux de Dumas (La Mole et Coconnas). Mais elle donne également, inscrite en bas de page, la mention des lieux et du temps de l'écriture de l'œuvre littéraire : "Portau-Prince, décembre 2002 - Kalat, $2005 »$. Ainsi se trouvent entrelacés, comme dans un jeu de miroirs se réfléchissant l'un l'autre, la démarche de l'écriture et les événements du réel.

\section{Le vrai au travers du prisme du surréel}

Le lecteur de Jérémie! Jérémie !, à l'instar de Fabrice Jaloux, doit ressortir transformé de sa lecture du livre, en particulier dans sa relation au réel. Il lui faut garder en mémoire cette réflexion de Fabrice dans le roman : « en Haïti c'est par la voie du surréel qu'on arrive au vrai » (p. 222). Au-delà du réel, et par le prisme du «surréel», c'est donc principalement «le vrai» que recherchent Fabrice et, à travers lui, Dominique Fernandez. Le voyage de Fabrice s'avère ainsi être une quête de vérité sur soimême et sur le monde, à laquelle le lecteur est convié à son tour. Fabrice entreprend une lutte exemplaire contre le mensonge, quelle qu'en soit la forme, et sa quête ne craint pas 
de passer par l'illusion - peut-être plus réelle que toute autre chose - des croyances, de la littérature et des arts. Ce combat pour la découverte du vrai ne se résout pour le héros que par la confrontation avec les autres, par la prise de conscience de sa véritable identité et par la découverte de son histoire personnelle. Ce combat aboutit finalement au sacrifice et à la mort du héros. Pour Dominique Fernandez, en effet, «les romans finissent toujours mal », car « découvrir la vérité, ça se paye toujours. ${ }^{6}$

\section{Bibliographie}

FERNANDEZ, Dominique. (2009), Ramon, Paris, Grasset;

- (2005), Jérémie! Jérémie!, Paris, Grasset;

-. (2004), Dictionnaire amoureux de la Russie, Paris, Plon;

-. (2003), La Course à l'abîme, Paris, Grasset;

-. (1980), Le Promeneur amoureux: de Venise à Syracuse, Paris, Plon;

- (1999), Les douze muses d'Alexandre Dumas, Paris, Grasset;

- et Ferrante FERRANTI. ([1997] 2007), Le Voyage d'Italie: Dictionnaire amoureux, Paris, Librairie académique Perrin;

- et Ferrante FerRanTI. ([1995] 1999), La Perle et le croissant: l'Europe baroque de Naples à Saint-Pétersbourg, Paris, Omnibus.

6 Propos tenus par Dominique Fernandez lors d'une interview réalisée en 2006 et diffusée sur le site des éditions Hachette. 


\title{
Résumé
}

Jérémie! Jérémie! est l'un des ouvrages les plus récents de Dominique Fernandez. Cette fiction évoque le séjour d'un jeune étudiant sur l'île d'Haïti, sa découverte du pays et les émois amoureux qu'il y ressentira. La description d'Haïti, par le prisme du héros romanesque, permet un jeu habile avec la géographie politique du lieu: mêlant réflexion historique, descriptions détaillées et aventures fictives, l'auteur parvient à créer un espace riche de vérité et de sens, où le réel acquiert de nouvelles perspectives par le double biais de la fiction romanesque et de l'observation précise. La transgression du réel, opérée par l'auteur, symbolise alors d'autres formes de subversion (politique, sociale, morale, sexuelle ou affective), observées ou effectuées par le héros, notamment par le biais de l'art et de l'écriture.

\begin{abstract}
Jérémie ! Jérémie ! is one of the latest works of the contemporary French author Dominique Fernandez. This novel is about the journey of a young student to Haiti, his discovery of the country and the new feelings he will experience there. Depicting Haiti through the eyes of Fabrice, the hero of the book, the novel provides us with a description of the local geography and politics. By blending historical relections on history, detailed descriptions and fictional adventures, the author succeeds in creating a truthful and meaningful fictional world, in which reality is endowed with new prospects through fiction and observation. So, going beyond reality, the author also points out to other forms of subversion, whether political, social, moral, sexual or affective. The hero will be able to witness and experience such forms of subversion on the island, mainly through art and writing.
\end{abstract}

